\title{
Editorial
}

\section{High-Tech Acupuncture and Integrative Laser Medicine 2013}

\author{
Gerhard Litscher, ${ }^{1,2}$ Xin-Yan Gao, ${ }^{2}$ Lu Wang, ${ }^{1}$ and Bing $\mathrm{Zhu}^{2}$ \\ ${ }^{1}$ Stronach Research Unit for Complementary and Integrative Laser Medicine, \\ Research Unit of Biomedical Engineering in Anesthesia and Intensive Care Medicine, TCM Research Center Graz, \\ Medical University of Graz, Auenbruggerplatz 29, 8036 Graz, Austria \\ ${ }^{2}$ Institute of Acupuncture and Moxibustion, China Academy of Chinese Medical Sciences, Beijing 100700, China \\ Correspondence should be addressed to Gerhard Litscher; gerhard.litscher@medunigraz.at
}

Received 1 November 2013; Accepted 1 November 2013

Copyright (C) 2013 Gerhard Litscher et al. This is an open access article distributed under the Creative Commons Attribution License, which permits unrestricted use, distribution, and reproduction in any medium, provided the original work is properly cited.

This is an annual special issue. The current issue is the 2013 issue which includes eleven interesting papers.

Acupuncture has been used for medical treatment for thousands of years. A large number of empirical data is available, but the technical quantification of effects was not possible up to now. Using electroacupuncture, needle, or laser stimulation and modern biomedical techniques, it was possible for the first time to quantify changes in biological activities caused by acupuncture.

As mentioned above, this special issue contains eleven publications, of which six are related to laser acupuncture, three to electroacupuncture, two to needle acupuncture, and one to photoluminescent bioceramic material. Apart from body acupuncture, special emphasis is also given to auricular acupuncture. One review article, 22 pages in length, deals with the topic auricular acupuncture with laser. The investigations cover animal experimental studies and studies in healthy volunteers, preterm neonates, and patients, as well as basic and clinical research on evidence-based high-tech acupuncture, integrative laser irradiation, and translational medicine.

It has to be mentioned that this annual special issue 2013 contains, among others, the following topics:

(i) modernization of acupuncture (evidence-based medicine, integrative laser medicine),

(ii) high-tech acupuncture,

(iii) development of innovative acupuncture stimulation methods, (iv) scientific evaluation of complementary medical methods (acupuncture, electroacupuncture, and low level laser therapy),

(v) clinical effects of acupuncture,

(vi) intravenous laser blood irradiation,

(vii) laser needle acupuncture,

(viii) red and infrared laser stimulation,

(ix) auricular acupuncture with laser,

(x) biomedical assessment of acupuncture,

Modernization of acupuncture is performed by high-tech acupuncture and integrative laser stimulation. Eastern and Western medicine are getting closer to each other using modern biomedical engineering technology, as described in this annual special issue.

\section{Conflict of Interests}

The authors state that there is no conflict of interests regarding the publication of this editorial.

\section{Acknowledgments}

As in the first annual special issue, the Lead Guest Editor again wishes to thank the other three Guest Editors, Professor Bing Zhu, (Director of the Institute of Acupuncture and Moxibustion, China Academy of Chinese Medical Sciences, Beijing, China), Associate Professor Xin-Yan Gao (Head of 
the Department of Physiology, Institute of Acupuncture and Moxibustion, China Academy of Chinese Medical Sciences, Beijing, China), and Professor Lu Wang (Stronach Research Unit for Complementary and Integrative Laser Medicine and TCM Research Center Graz, Medical University of Graz, Graz, Austria), for the excellent cooperation. In this context, I would also like to thank all authors for their excellent contributions and patience during the review process. The work of all reviewers on the papers within this special issue is highly appreciated.

We want to thank Ms. Ingrid Gaischek (Stronach Research Unit for Complementary and Integrative Laser Medicine, Research Unit of Biomedical Engineering in Anesthesia and Intensive Care Medicine, and TCM Research Center Graz, Medical University of Graz, Graz, Austria) for her valuable support in every respect.

\author{
Gerhard Litscher \\ Xin-Yan Gao \\ Lu Wang \\ Bing Zhu
}




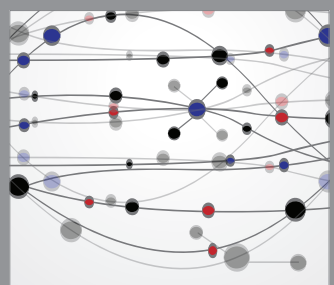

The Scientific World Journal
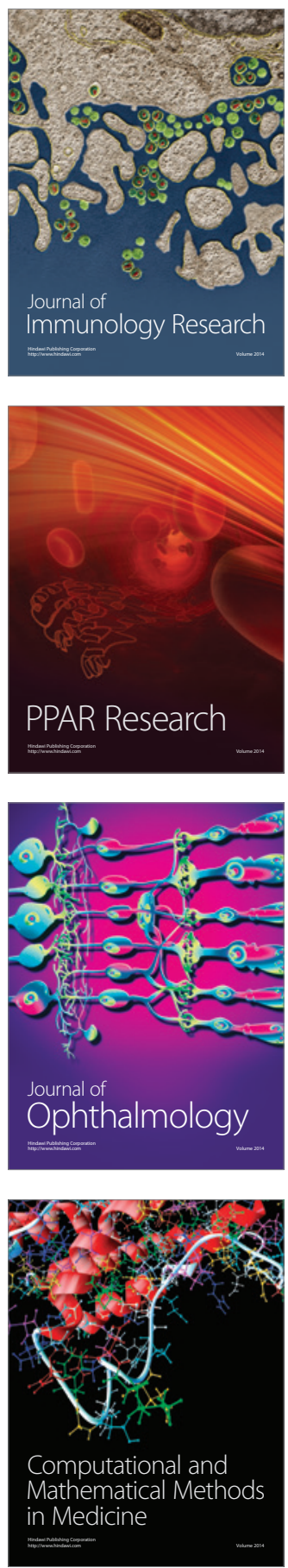

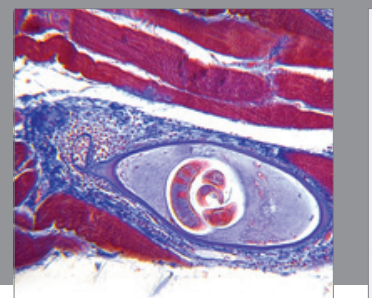

Gastroenterology

Research and Practice
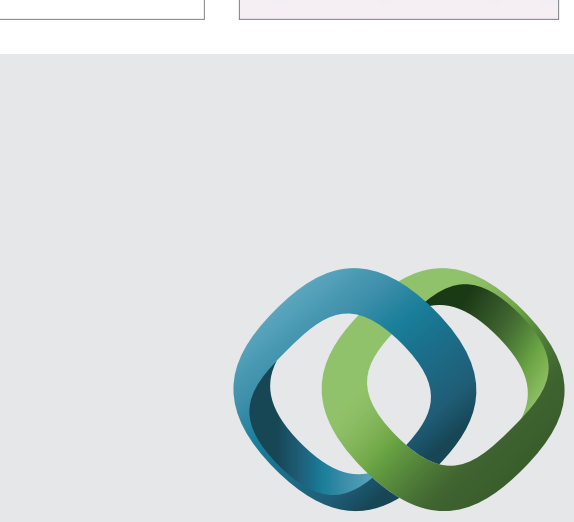

\section{Hindawi}

Submit your manuscripts at

http://www.hindawi.com
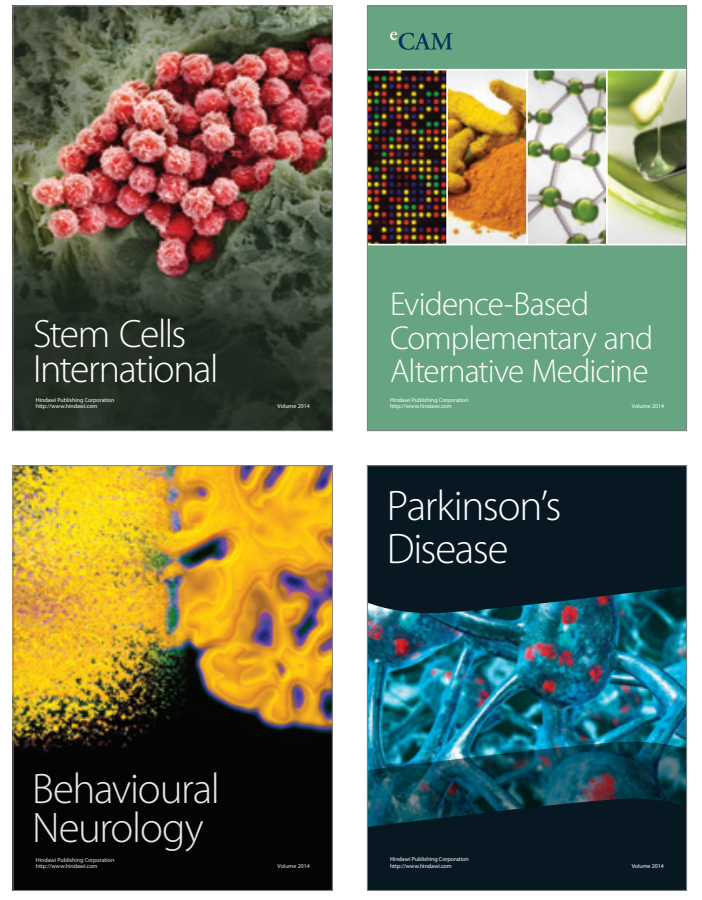
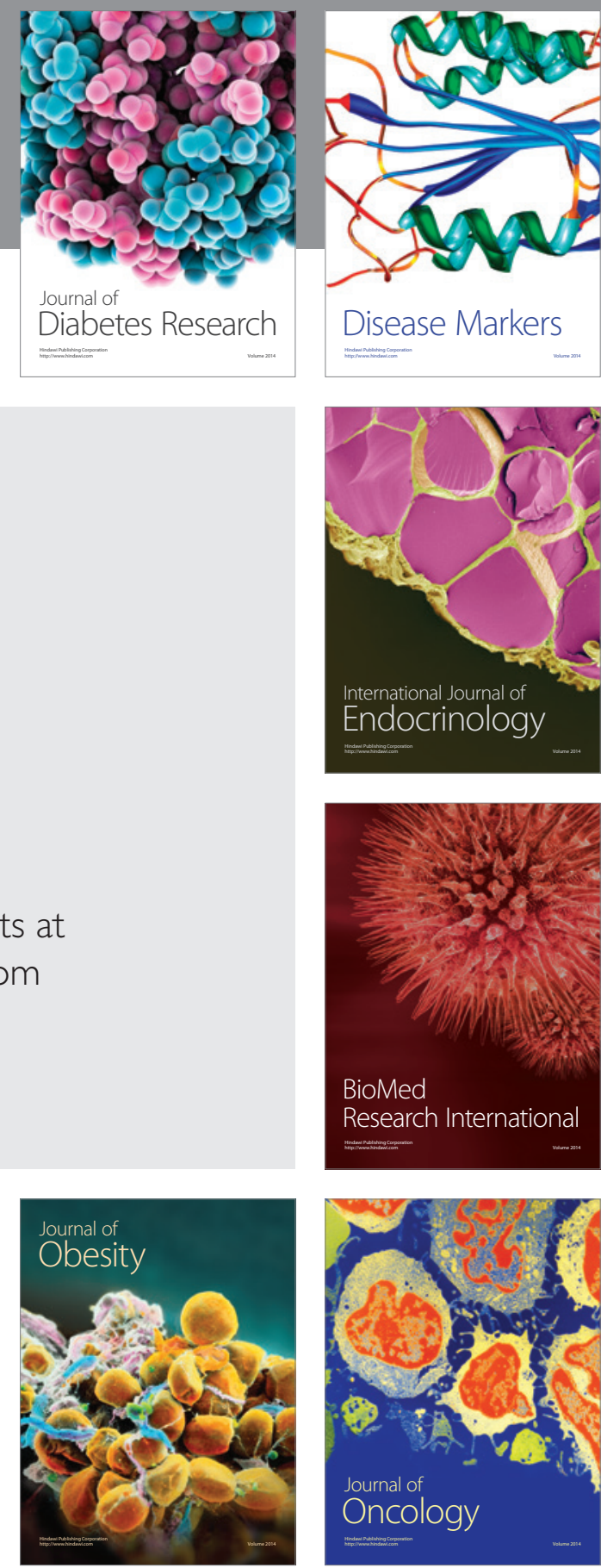

Disease Markers
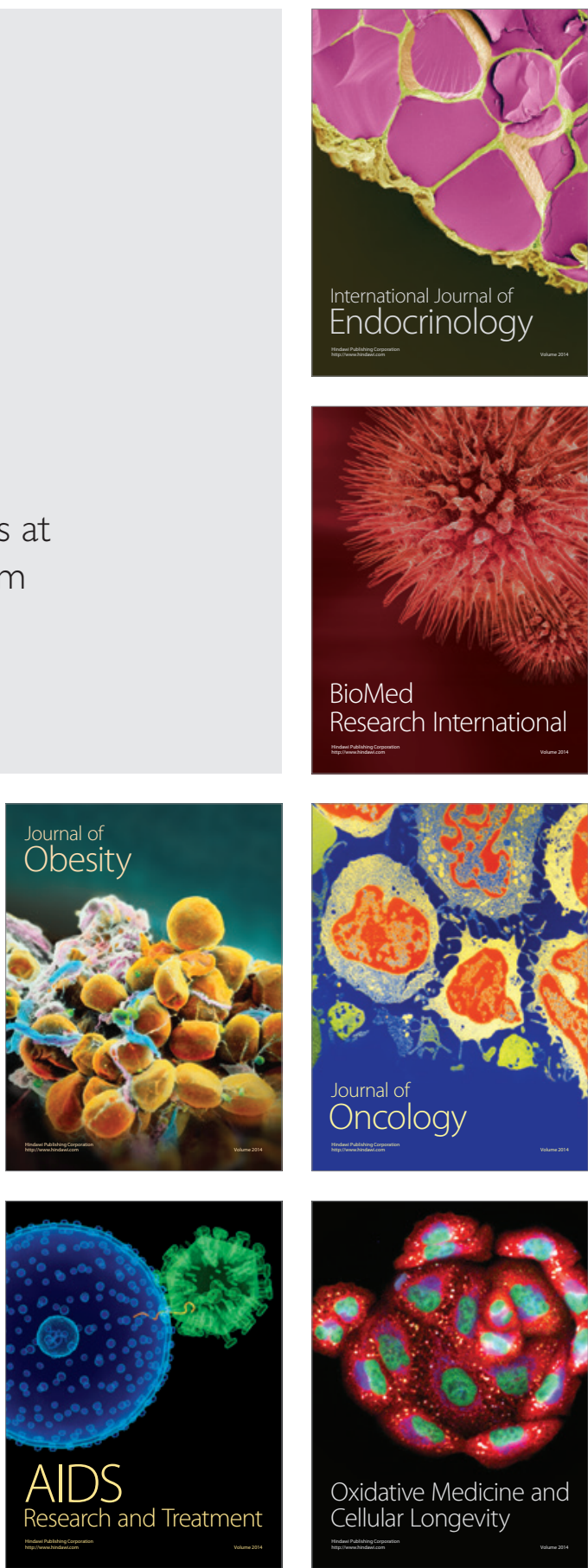